\title{
Bit Error Rate Estimation Methods for QPSK CO-OFDM Transmission
}

\author{
Son Thai Le, Keith J. Blow, Vladimir K. Mezentsev and Sergei K. Turitsyn
}

\begin{abstract}
Coherent optical orthogonal frequency division multiplexing (CO-OFDM) is an attractive transmission technique to virtually eliminate inter-symbol interference caused by chromatic dispersion and polarization-mode dispersion. Design, development, and operation of CO-OFDM systems require simple, efficient and reliable methods of their performance evaluation. In this paper, we demonstrate an accurate bit error rate estimation method for QPSK CO-OFDM transmission based on the probability density function of the received QPSK symbols. By comparing with other known approaches, including data-aided and nondata-aided EVM, we show that the proposed method offers the most accurate estimate of the system performance for both single channel and wavelength division multiplexing QPSK CO-OFDM transmission systems.
\end{abstract}

Index Terms - Bit error rate, coherent detection, coherent optical transmission, orthogonal frequency division multiplexing.

\section{INTRODUCTION}

$\mathrm{C}$ OHERENT optical orthogonal frequency division multiplexing (CO-OFDM) is considered as a promising candidate for future long-haul high capacity transmission systems [1]. CO-OFDM provides an efficient way to compensate for inter-symbol interference caused by both chromatic dispersion (CD) and polarization-mode dispersion (PMD) $[2,3]$. In addition, CO-OFDM also offers flexibility in accessing individual subcarriers in a multi-user environment, and a simplified equalization scheme [4]. The design, development, and operation of CO-OFDM systems all require simple, efficient and reliable methods of their performance evaluation.

The bit error rate (BER) in CO-OFDM systems can be estimated in numerical investigations using Monte Carlo simulation and in experiments (typically with off-line signal processing) by directly counting the number of errors at the receiver. The corresponding Q-factor is calculated using the inverse complementary error function [5]. However, this method relies on a large number of statistical samples and, in general, is time-consuming, especially if the signal quality is

Manuscript received November 08, 2013. S. T. Le M, K. J. Blow, V. K Menzentsev and S. K. Turitsyn are with Aston Institute of Photonic Technologies (AIPT), School of Engineering and Applied Science, Aston Triangle, Birmingham, B4 7ET, UK (corresponding author phone: +44(0)744-702-40-09; e-mail: let1@aston.ac.uk). Copyright (c) 2013 IEEE. Personal use of this material is permitted. However, permission to use this material for any other purposes must be obtained from the IEEE by sending a request to pubs-permissions@ieee.org. high and massive optimization modelling is required. It is highly desirable and practically important to develop efficient indirect numerical and statistical methods for evaluating $\mathrm{CO}$ OFDM system performance.

For coherent communication systems with multi-level signals both in amplitude and in phase, the error vector magnitude (EVM) is commonly used as a fast measure of the received digital signal's quality $[6,7]$. The EVM describes the effective distance of the received complex symbol from its ideal position in the constellation diagram. In an additive white Gaussian noise (AWGN) transmission channel the connection of EVM to BER can be determined theoretically [7]. The standard EVM is a data-aided estimation technique, where for measurement purposes the transmitted data are known [6]. On the other hand, it is more common for real-world receivers that the sent data (e.g. training sequences) are unknown. In this case, nondata-aided reception can be applied as shown in [8]. Several other relevant methods of evaluating the signal quality have recently been proposed, experimentally verified and compared for single carrier QPSK systems [9-11]. However, the exact relationship between the BER and the EVM in COOFDM still remains an open problem. In addition, the relative performances of different BER estimation methods for coherent QPSK systems have to be examined carefully when being applied for CO-OFDM transmission.

Recently, we have proposed a novel statistical BER estimation method for CO-OFDM transmissions [12] based on the probability density function of the received QPSK symbols. The proposed BER estimation method was evaluated in comparison with other known approaches for single channel $112 \mathrm{~Gb} / \mathrm{s}$ polarization division multiplexing (PDM) COOFDM transmissions with a cyclic prefix (CP) in [12]. In this paper, we extend our previous work [12] by studying the statistical properties of QPSK signals and demonstrating the effectiveness of this method in reduced-guard-interval (RGI) and wavelength division multiplexing (WDM) CO-OFDM transmissions. In addition, the robustness of the proposed BER estimation method to laser phase noise and frequency offset is also discussed.

\section{BER ESTIMATION METHODS FOR OPTICAL QPSK TRANSMISSIONS}

\section{A. Direct Error-Counting}

The BER can be directly measured by counting the number 
of errors at the receiver subject to sufficient symbols being recorded. The measured (or estimated) BER is usually converted to an equivalent "Gaussian noise" $\mathrm{Q}$-factor in $\mathrm{dB}$ using the expression:

$$
Q_{B E R}=20 \log \left[\sqrt{2} \cdot \operatorname{efrc}^{-1}(2 B E R)\right]
$$

Where $e r f c^{-1}$ is the inverse complementary error function. This sets the reference Q-factor used in the following evaluation of different indirect methods.

\section{B. Data-aided EVM}

In an optical communication system with QPSK modulation format, the data is encoded in the phase of the optical electrical field. The complex amplitude of this field can be described by 4 points in a complex constellation plane. At the receiver, after propagation through the fiber link, the received signal vector $\mathbf{E}_{\mathrm{r}}$ deviates by an error vector $\mathbf{E}_{\mathrm{err}}$ from the ideal transmitted vector $\mathbf{E}_{\mathrm{t}}$ as shown in Fig. 1. The data-aided EVM is defined by a root mean square of $\mathbf{E}_{\text {err }}$ and embraces all (linear and nonlinear) impairments [6]:

$$
E V M_{m}=\frac{\sigma_{e r r}}{\left|\boldsymbol{E}_{t, m}\right|}, \sigma_{e r r}^{2}=\left\langle\left|\boldsymbol{E}_{e r r, i}\right|^{2}\right\rangle, \boldsymbol{E}_{e r r, i}=\boldsymbol{E}_{r, i}-\boldsymbol{E}_{t, i}
$$

where $\langle\cdot\rangle$ stands for the averaging operation, $\mathbf{E}_{\mathrm{t}, \mathrm{m}}$ is the longest ideal constellation vector, serving for normalization.

By applying the definition (2), the EVM in QPSK COOFDM transmissions can be calculated as:

$$
E V M=\frac{\sqrt{\left\langle\left|c_{k}-c_{k, \text { ideal }}\right|^{2}\right\rangle}}{\left|c_{\text {ideal }}\right|}
$$

where $c_{k}$ is the $k^{\text {th }}$ received symbol and $c_{k \text {,ideal }}$ is the corresponding ideal constellation point. Note that for QPSK signals all ideal constellation points are allocated in a circle: $\left|c_{k, \text { ideal }}\right|=\left|c_{\text {ideal }}\right|$.

For a QPSK system with AGWN channel the BER can be estimated from the EVM as [7]:

$$
B E R=\frac{1}{2} \operatorname{erfc}\left(\frac{E V M^{-1}}{\sqrt{2}}\right)
$$

By substituting (4) into (1), we can define the equivalent Qfactor in $\mathrm{dB}$ knowing the EVM as:

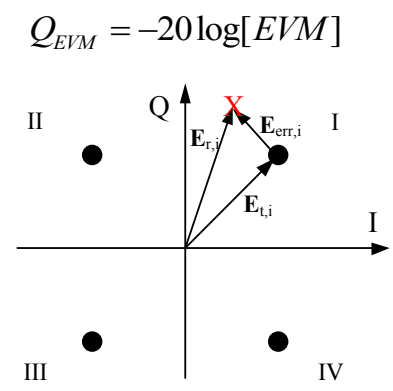

Fig. 1. Constellation diagram and error vector for a QPSK signal. Ideal constellation diagram with a received value $\mathrm{X}$. Vector $\mathbf{E}_{\mathrm{t}, \mathrm{i}}$ is the transmitted signal, vector $\mathbf{E}_{\mathrm{r}, \mathrm{i}}$ is the received signal and $\mathbf{E}_{\mathrm{err}, \mathrm{i}}=\mathbf{E}_{\mathrm{r}, \mathrm{i}}-\mathbf{E}_{\mathrm{t}, \mathrm{i}}$ is the error vector.

\section{Nondata-aided EVM (Q factor 1, Q1)}

The EVM can also be calculated without knowing the transmitted data. The most common approach for calculating nondata-aided EVM is to perform hard decision on the received symbols and then apply the expression (2) [6]. In this case, the error vector of a received symbol is calculated according to the nearest ideal constellation point. As a consequence, nondata-aided EVM tends to under-estimate the EVM if the received signal is strongly noisy.

Another nondata-aided EVM has been proposed for QPSK CO-OFDM transmission in [8]. In this technique the EVM is calculated by replacing the four ideal QPSK constellation points with the mean values of the received symbols in the four quadrants of the constellation diagram:

$$
E V M 1=\sqrt{\left\langle\left|c_{k, i}-c_{\text {avg }, i}\right|^{2} /\left|c_{a v g, i}\right|^{2}\right\rangle}
$$

where $c_{\text {avg,i }}, i=1,2,3,4$ are the means of the received symbols $c_{k, i}$ that fall into the i'th quadrant of the constellation diagram. For comparison purpose we also convert this nondata-aided EVM into an equivalent Q-factor in $\mathrm{dB}$ by the expression:

$$
Q 1=-20 \log [E V M 1]
$$

\section{Q-factor $2\left(Q^{2}\right)$}

It has been shown [13] that for single carrier QPSK systems without optical dispersion compensation, the four components of a QPSK signal (in-phase x-polarization, in-phase $y$ polarization, quadrature $\mathrm{x}$-polarization, quadrature $\mathrm{y}$ polarization) are Gaussian distributed (or at least nearly Gaussian distributed) and statistically independent both before and after the digital signal processing (DSP) in the receiver [13]. Therefore, a QPSK constellation can be decomposed into two binary-phase-shift-keying (BPSK) constellations (0 and $\pi$ ), or equivalently two amplitude-shift-keying (ASK) constellations ( 1 and -1), for the in-phase and quadrature components [5]. As a result, following the same well known approach for calculating the conventional Q-factor for on-offkeying (OOK) signals, we can define the Q-factors of the inphase and quadrature components of the received QPSK signals by [9]:

$$
\begin{gathered}
Q_{\mathrm{Re}}=\frac{\left\langle c_{k, \mathrm{Re}}\left(c_{k, \mathrm{Re}}>0\right)\right\rangle-\left\langle c_{k, \mathrm{Re}}\left(c_{k, \mathrm{Re}}<0\right)\right\rangle}{\sigma_{\mathrm{Re}}\left(c_{k, \mathrm{Re}}>0\right)+\sigma_{\mathrm{Re}}\left(c_{k, \mathrm{Re}}<0\right)} \\
Q_{\mathrm{Im}}=\frac{\left\langle c_{k, \mathrm{Im}}\left(c_{k, \mathrm{Im}}>0\right)\right\rangle-\left\langle c_{k, \mathrm{Im}}\left(c_{k, \mathrm{Im}}<0\right)\right\rangle}{\sigma_{\mathrm{Im}}\left(c_{k, \mathrm{Im}}>0\right)+\sigma_{\mathrm{Im}}\left(c_{k, \mathrm{Im}}<0\right)}
\end{gathered}
$$

where $\sigma(\cdot)$ denotes the standard deviation (STD) of the statistical samples. In (8) and (9) $\mathrm{Q}_{\mathrm{Re}}\left(\mathrm{Q}_{\mathrm{Im}}\right)$ is calculated as the ratio between the difference of the means and the sum of the STDs of in-phase (quadrature) components with opposite signs. The BER then can be obtained by using the estimations from both in-phase and quadrature components:

$$
B E R=\left\langle\left[\frac{1}{2} \operatorname{erfc}\left(\frac{Q_{\mathrm{Re}}}{\sqrt{2}}\right), \frac{1}{2} \operatorname{erfc}\left(\frac{Q_{\mathrm{Im}}}{\sqrt{2}}\right)\right]\right\rangle
$$




\section{E. $Q$-factor $3(Q 3)$}

Another definition of Q-factor was introduced in [9] as the ratio between the mean and the STD value of each constellation point. For the symbol in the first quadrant, the Qfactors are:

$$
\begin{gathered}
Q_{1, \mathrm{Re}}=\frac{\left|\left\langle c_{k, \mathrm{Re}}\left(c_{k, \mathrm{Re}}>0, c_{k, \mathrm{Im}}>0\right)\right\rangle\right|}{\sigma_{\mathrm{Re}}\left(c_{k, \mathrm{Re}}>0, c_{k, \mathrm{Im}}>0\right)} \\
Q_{1, \mathrm{Im}}=\frac{\left|\left\langle c_{k, \mathrm{Im}}\left(c_{k, \mathrm{Re}}>0, c_{k, \mathrm{Im}}>0\right)\right\rangle\right|}{\sigma_{\mathrm{Im}}\left(c_{k, \mathrm{Re}}>0, c_{k, \mathrm{Im}}>0\right)}
\end{gathered}
$$

The overall BER can be obtained by using $\mathrm{Q}_{\mathrm{i}, \mathrm{Re}}$ and $\mathrm{Q}_{\mathrm{i}, \mathrm{Im}}$, $\mathrm{i}=1,2,3,4$ of all the constellation symbols [9-11]:

$$
B E R=\left\langle\left[\frac{1}{2} \operatorname{erfc}\left(\frac{Q_{i, \mathrm{Re}}}{\sqrt{2}}\right), \frac{1}{2} \operatorname{erfc}\left(\frac{Q_{i, \mathrm{Im}}}{\sqrt{2}}\right)\right]\right\rangle
$$

\section{Simulation Setup of 112 GB/s QPSK CO-OFDM TRANSMISSIONS}

For investigating the statistical properties of QPSK signals and comparing the performances of different BER estimation methods, we set up a $112 \mathrm{~Gb} / \mathrm{s}$ PDM CO-OFDM transmission system, the block diagram of which is shown in Fig. 2.

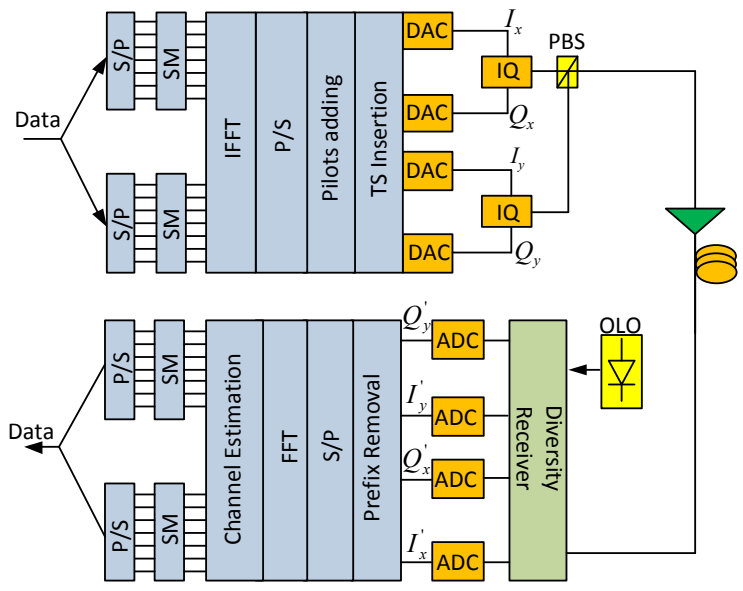

Fig. 2. Block diagrama of $112 \mathrm{~Gb} / \mathrm{s}$ PDM CO-OFDM transmissions. S/P: serial/parallel conversion, $\mathrm{P} / \mathrm{S}$ : parallel/serial conversion, $\mathrm{SM}$ : symbol mappings, TS: training symbol, DAC: digital-to-analog converter, I/Q: I/Q modulator, OLO: optical local oscilator

The data stream is first divided into $\mathrm{x}$ - and $\mathrm{y}$-polarizations, each of which is then mapped onto 2048 subcarriers using QPSK modulation format with Gray code and subsequently transferred to the time domain by an IFFT of size 4096 while zeros occupy the remainder for oversampling purpose. The OFDM useful duration is $60 \mathrm{~ns}$. A cyclic prefix (CP) of length $12 \mathrm{~ns}$ is used to accommodate dispersion. The long-haul fiber link is assumed to consist of $80-\mathrm{km}$ spans of standard single mode fiber (SSMF) with the loss parameter of $0.2 \mathrm{~dB} / \mathrm{km}$, nonlinearity coefficient of $1.22 \mathrm{~W}^{-1} \mathrm{~km}^{-1}$, dispersion of $16 \mathrm{ps} / \mathrm{nm} / \mathrm{km}$ and PMD coefficient of $0.1 \mathrm{ps} / \mathrm{km}^{0.5}$. The fiber span loss is compensated by Erbium-doped fiber amplifiers (EDFA) with $16 \mathrm{~dB}$ of gain and a noise figure of $6 \mathrm{~dB}$. In the simulation amplified spontaneous emission (ASE) noise is added inline. The transmitter and receiver lasers have the same linewidth of $100 \mathrm{kHz}$. The laser phase noise is modeled as a Wiener-Levy process with a variance $\sigma^{2}=2 \pi v t$ where $v$ is the combined laser linewidth and $t$ is the time difference between two samples [14]. The simulated time window contains 100 OFDM symbols (409600 bits). The channel estimation and equalization (including polarization demultiplexing and channel response equalization) is done with the assistance of an initial training sequence ( 2 OFDM symbols in each polarization) using the zero forcing estimation method with MIMO processing [15]. The common phase error (CPE) due to laser phase noises is estimated and compensated using the pilot-aided technique by inserting 16 pilot subcarriers in each OFDM symbol. In the simulation the timing synchronization is assumed to be perfect. Furthermore, the frequency offset between transmitter and receiver lasers was not considered (except section VIII).

Another CO-OFDM configuration known as reduced-guardinterval CO-OFDM [16] is also considered here. In RGI COOFDM transmissions a short $\mathrm{CP}$ is added to each OFDM symbol to accommodate the inter-symbol interference (ISI) with short memory, such as fiber PMD or residual chromatic dispersion. The accumulated dispersion of the optical link is compensated at the receiver using overlapped frequencydomain equalizers (OFDE) [17, 18] or time domain finite impulse response (FIR) filters $[19,20]$. In this work the OFDE with overlap-save method was applied [17]. As the chromatic dispersion can be effectively compensated at the receiver, a shorter symbol duration can be used in RGI CO-OFDM. As a result, in RGI CO-OFDM transmissions a smaller number of subcarriers can be used [16]. We consider a $112 \mathrm{~Gb} / \mathrm{s}$ RGI CO-OFDM transmission system with 112 subcarriers. The useful OFDM symbol duration is $3.8 \mathrm{~ns}$ and the CP length is $0.2 \mathrm{~ns}$. In applying OFDE with overlap-save method, a block size of 10 OFDM symbols (40 ns) was used.

\section{Statistical PROPERTIES OF ReCEIVED QPSK SYMBOLS IN CO-OFDM TRANSMISSIONS}

For single carrier PDM QPSK systems in uncompensated links, the statistical properties of the received nonlinear interference noise have been investigated and compared with different modulation formats in $[13,21]$. In this section, we study in detail for the first time the statistical properties of QPSK signal in PDM CO-OFDM transmissions. We take into account not only the four aforementioned components but also the phase of the QPSK signal. The simulation setup of a $112 \mathrm{~Gb} / \mathrm{s}$ QPSK PDM CO-OFDM system is discussed in detail in the previous section.

The histograms of in-phase and quadrature components of QPSK signal in $112 \mathrm{~Gb} / \mathrm{s}$ PDM CO-OFDM transmissions are shown in Fig. 3 and Fig. 4 (only for x-polarization, a similar result for y-polarization is observed, but not shown here) for different values of the launch power $(3 \mathrm{dBm}$ and $6 \mathrm{dBm})$. The Gaussian fitting is obtained by calculating the mean and STD of the received statistical samples. In this simulation the amplified spontaneous emission (ASE) noise added by erbium- 
doped fiber amplifiers (EDFA) is not considered in order to analyze the distribution of signal components alone. In addition, the transmitter and receiver lasers are considered as noiseless. The only source of noise is the fiber nonlinearity. We find that the distributions of in-phase and quadrature components of QPSK signals are Gaussian-like only for small values of the launch power. Herein, the well-known Kolmogorov-Smirnov test (K-S test) was applied to define if a statistical signal has a Gaussian-like distribution. The Kolmogorov-Smirnov statistic (KSSTAT) for a given cumulative distribution function $F(x)$ is defined as:

$$
D=\sup \left|F_{n}(x)-F(x)\right|
$$

where $F_{n}(x)$ is the empirical distribution function for $n$ observations of the statistical signal. The typical value of the KSSTAT for a Gaussian-like signal is below 0.05 .

If the launch power is set to $3 \mathrm{dBm}$ (no errors were detected at the receiver, 409600 bits were sent) a small mismatch between the actual distribution and its Gaussian fitting can be observed (Fig. 3). If the launch power is increased to $6 \mathrm{dBm}$ $(\mathrm{BER}=0.0002)$ the mismatch becomes obvious (Fig. 4) and the Gaussian distribution shows a poor approximation of the distribution of in-phase and quadrature components of the received QPSK symbols.
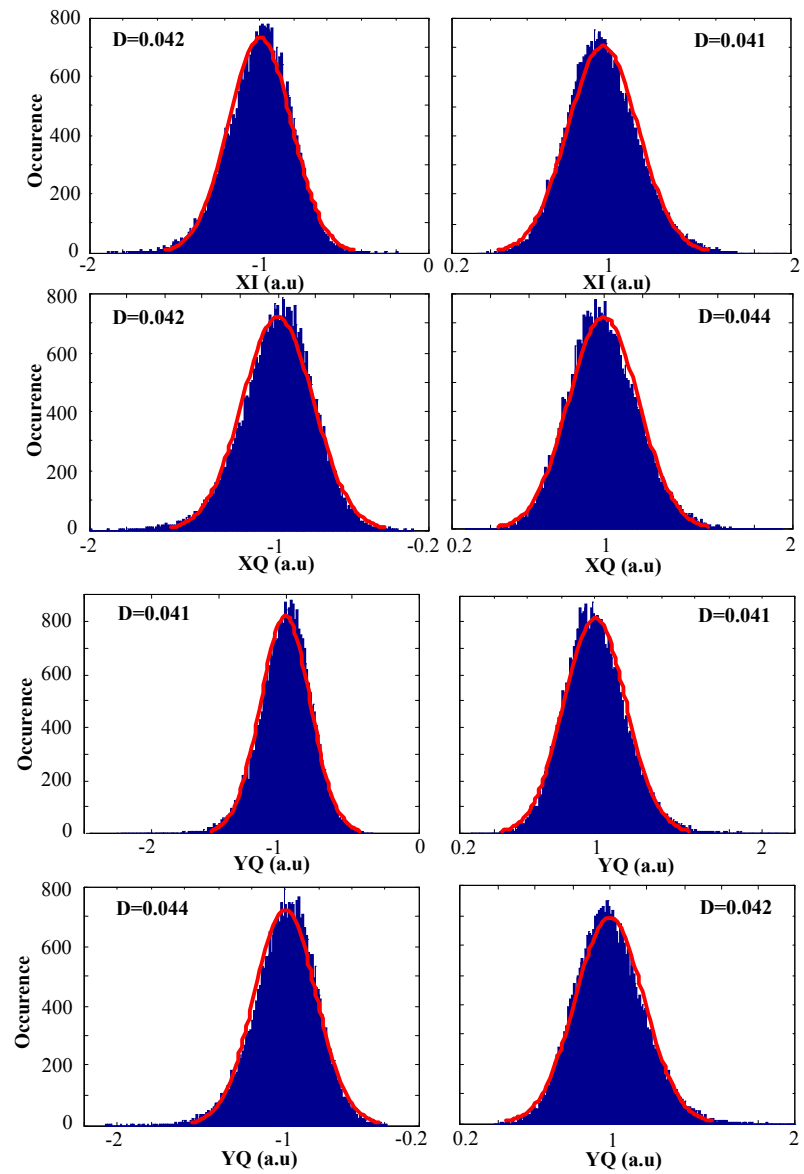

Fig. 3. Histogram of in-phase and quadrature components of the received QPSK symbols in $\mathrm{x}$ and y-polarization. Propagation over $800 \mathrm{~km}$ in nonlinear limited regime $(3 \mathrm{dBm})$. Gaussian fitting is superimposed to each histogram, KSSTAT values are also included in each histogram.
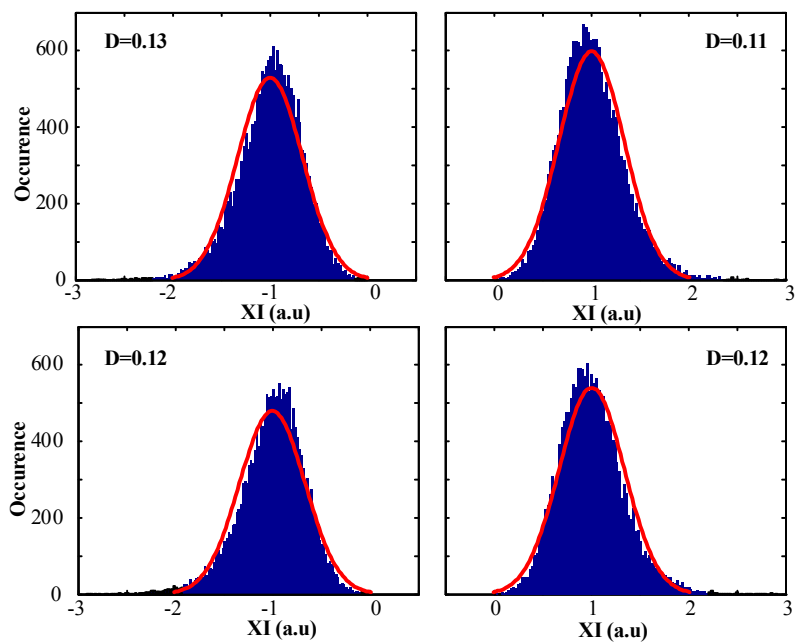

Fig. 4. Histogram of in-phase and quadrature components of the received QPSK symbols in x-polarization. Propagation over $800 \mathrm{~km}$ in nonlinear limited regime with the launch power of $6 \mathrm{dBm}$.
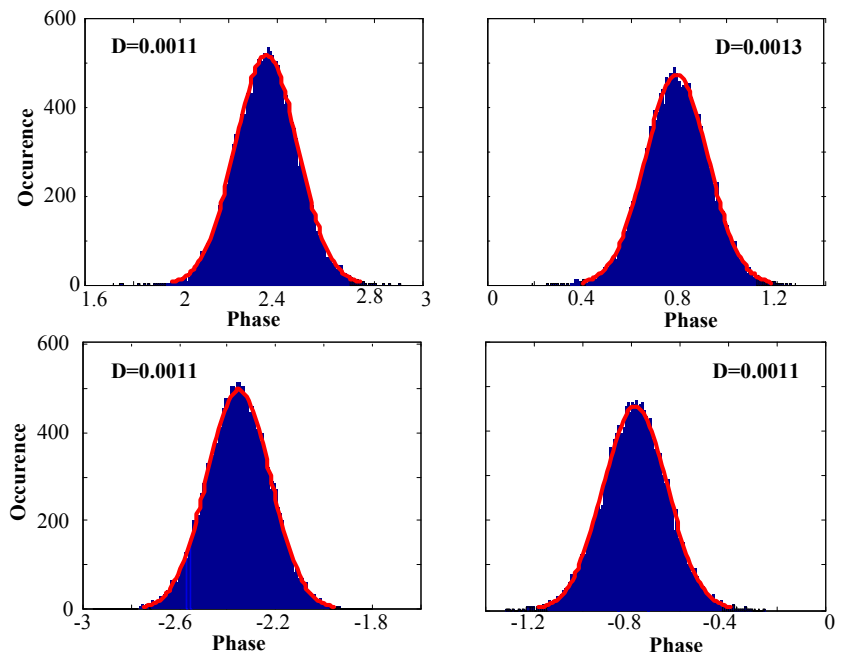

Fig. 5. Histogram of the received QPSK symbols' phases (x-polarization) in four quadrants of the constellation diagram. Propagation over $800 \mathrm{~km}$ in nonlinear limited regime with the launch power of $3 \mathrm{dBm}$.
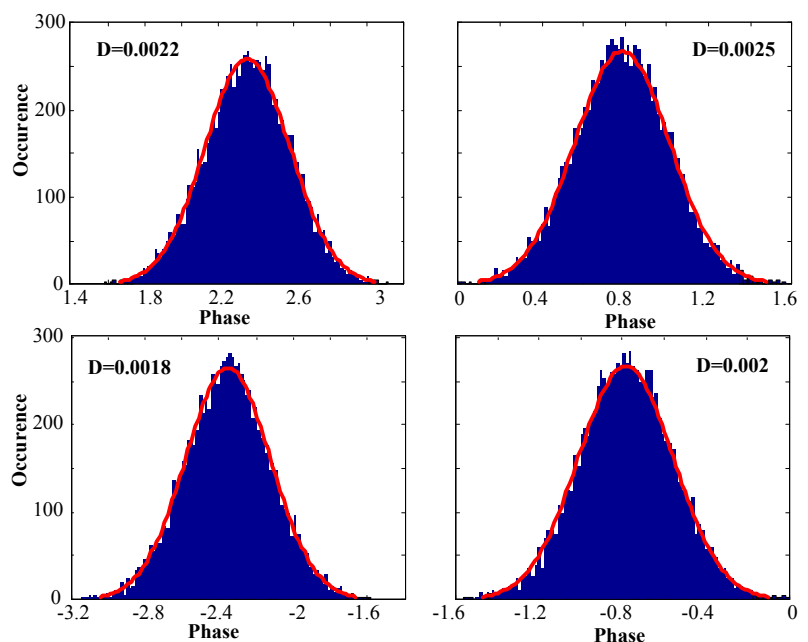

Fig. 6. Histogram of the received QPSK symbols' phases (x-polarization) in four quadrants of the constellation diagram. Propagation over $800 \mathrm{~km}$ in nonlinear limited regime with the launch power of $6 \mathrm{dBm}$. 
This result is different from what has been observed for single carrier QPSK transmissions in [13], showing that at high values of the launch power the nonlinear interference noise (NLIN) in CO-OFDM transmission deviates from Gaussian distribution. The obtained result herein agrees well with a recent study on the statistical property of NLIN in CO-OFDM transmission [22], indicating that the Gaussian assumption of NLIN, which is the key in the derivation of closed-form expression for the nonlinear performance of CO-OFDM in $[23,24]$ is, in general, not satisfied.

In addition, the statistical properties of in-phase and quadrature components of the received QPSK symbols in COOFDM transmissions are also sensitive to the specific DSP technique used, especially the CPE estimation and compensation. The CPE due to laser phase noise and fiber nonlinearity rotates the constellation diagram and thus changes the statistical properties of the in-phase and quadrature components significantly. In the presence of CPE offset due to the estimation inaccuracy, which usually occurs in the nonlinear limited regime, the PDF of in-phase and quadrature components cannot be approximated accurately by a Gaussian distribution (Fig. 4). As a consequence, the two aforementioned "Gaussian assumption" based BER estimation methods (expressions (10) and (13)) may not be effective for QPSK CO-OFDM transmissions.

Herein, we study the statistical properties of the phases of the received QPSK symbols. The histograms of the received QPSK symbols' phases (x-polarization) in four quadrants of the constellation diagram are shown in Fig. 5 and Fig. 6 for different values of the launch power $(3 \mathrm{dBm}$ and $6 \mathrm{dBm})$. We find that the distribution of the received QPSK symbols' phases in each quadrant of the constellation diagram is essentially Gaussian $(\mathrm{D}<0.05)$, independent of the launch power and the transmission distance. For the investigated system our analysis is carried out by changing the launch power from $-9 \mathrm{dBm}$ to $9 \mathrm{dBm}$ (in $3 \mathrm{~dB}$ steps) and the transmission distance from 400 to $2400 \mathrm{~km}$ (in $400 \mathrm{~km}$ steps). This phenomenon can be explained by the fact that the nonlinear phase noise in CO-OFDM transmission is dominated by four-wave mixing (FWM) and its interaction with ASE noise. As a result, when independent data is carried on a large number of subcarriers, the central limit theorem can be applied for the nonlinear phase noise. In addition, the distribution of the received QPSK symbols' phases in each quadrant is nearly insensitive to the CPE offset, especially when the number of subcarriers is large, as the CPE offset affects only the mean value but not the STD of the received QPSK symbols' phases. As a result, a more reliable statistical BER estimation method for QPSK CO-OFDM transmissions can be developed based on the statistical properties of the QPSK symbols' phases.

\section{Proposed BER Estimation MEthod For QPSK CO- OFDM TRANSMISSIONS}

It has been shown in the previous section that the distribution of the received QPSK symbols' phases in each quadrant of the constellation diagram is in good agreement with a Gaussian distribution. Using a Gaussian approximation, the probability density function (PDF) of the received QPSK symbols' phases in four constellation quadrants can be expressed as:

$$
f_{k}(\phi)=\frac{1}{\sigma_{k} \sqrt{2 \pi}} \cdot \exp \left(-\frac{\left(\phi-\phi_{k}\right)^{2}}{2 \sigma_{k}^{2}}\right)
$$

where $f_{k}(\phi), \phi_{k}$ and $\sigma_{k}$ denote the PDF, means and standard deviations of the received phases in the $\mathrm{k}^{\text {th }}$ quadrant $(k=1,2,3$, 4). In QPSK (Gray coded) CO-OFDM systems, information symbols can have one of the four following values:

$X_{1}=\sqrt{2} \exp (j \pi / 4), X_{2}=\sqrt{2} \exp (j 3 \pi / 4)$,

$X_{3}=\sqrt{2} \exp (-j 3 \pi / 4), X_{4}=\sqrt{2} \exp (-j \pi / 4)$.

The error probability when $X_{I}$ is transmitted can be calculated as follows:

$$
\begin{aligned}
& P_{E}\left(X_{1}\right)=\int_{-\infty}^{0} f_{1}(\phi) d \phi+\int_{\pi / 2}^{+\infty} f_{1}(\phi) d \phi= \\
& =\frac{1}{2}\left[\operatorname{erfc}\left(\frac{\phi_{m, 1}}{\sigma_{1} \sqrt{2}}\right)+\operatorname{erfc}\left(\frac{\pi / 2-\phi_{m, 1}}{\sigma_{1} \sqrt{2}}\right)\right]
\end{aligned}
$$

Similarly, we can obtain expressions for $P_{E}\left(X_{2}\right), P_{E}\left(X_{3}\right)$, $P_{E}\left(X_{4}\right)$, then the system's BER is given by:

$$
B E R=\frac{1}{8} \sum_{k=1}^{4}\left[\operatorname{erfc}\left(\frac{\overline{\phi_{k}}-\theta_{k}+\pi / 4}{\sigma_{k} \sqrt{2}}\right)+\operatorname{erfc}\left(\frac{\theta_{k}+\pi / 4-\overline{\phi_{k}}}{\sigma_{k} \sqrt{2}}\right)\right]
$$

where $\theta_{k}=\arg \left(X_{k}\right), \quad k=1,2,3,4$. This expression offers a relatively simple way to estimate the performance of a $\mathrm{CO}$ OFDM system by calculating the means and STDs of the received phases in each quadrant of the constellation diagram. This BER estimation method is nondata-aided.

The proposed BER estimation method can also be extended for m-PSK CO-OFDM transmission as:

$$
B E R=\frac{1}{2 m} \sum_{k=1}^{m}\left[\operatorname{erfc}\left(\frac{\overline{\phi_{k}}-\theta_{k}+\pi / m}{\sigma_{k} \sqrt{2}}\right)+\operatorname{erfc}\left(\frac{\theta_{k}+\pi / m-\bar{\phi}_{k}}{\sigma_{k} \sqrt{2}}\right)\right]
$$

where $\theta_{k}=(2 k+1) \pi / m, k=0,1 \ldots m-1$ are the phases of m-PSK information symbols.

\section{Performance Comparison of Ber Estimation METHODS FOR QPSK CO-OFDM TRANSMISSIONS}

In this section we compare the performances of different BER estimation methods, namely data-aided EVM, nondataaided EVM (Q-factor 1), Q-factor 2, Q-factor 3 and the proposed method (expression (16)), for QPSK PDM COOFDM and 8-PSK PDM CO-OFDM transmissions. For comparison purposes the estimated BERs for $\mathrm{x}$ - and $\mathrm{y}$ polarization are averaged and then converted to a Q-factor using the expression (1).

The investigated BER estimation methods for $112 \mathrm{~Gb} / \mathrm{s}$ QPSK PDM CO-OFDM are compared in Fig. 7. The blue line with circle markers $(\mathrm{Q}(\mathrm{BER}))$ is the reference result of the direct error counting from Monte Carlo simulations (10 runs). The red line with square markers (Q-proposed) shows the result obtained using the estimation method proposed here based on a Gaussian approximation of the phase noise 
statistics (expression (17)). In Fig. 7 almost no mismatch between Q(BER) and Q-proposed is observed. This result indicates that the proposed BER estimation method is highly accurate. On the other hand, all the other BER estimation methods, namely EVM (data-aided, nondata-aided), Q-factor 2 and Q-factor 3, underestimate the system performance by approximatelly $1 \mathrm{~dB}$. Interestingly, all these BER estimation methods show almost the same performance for CO-OFDM transmission.

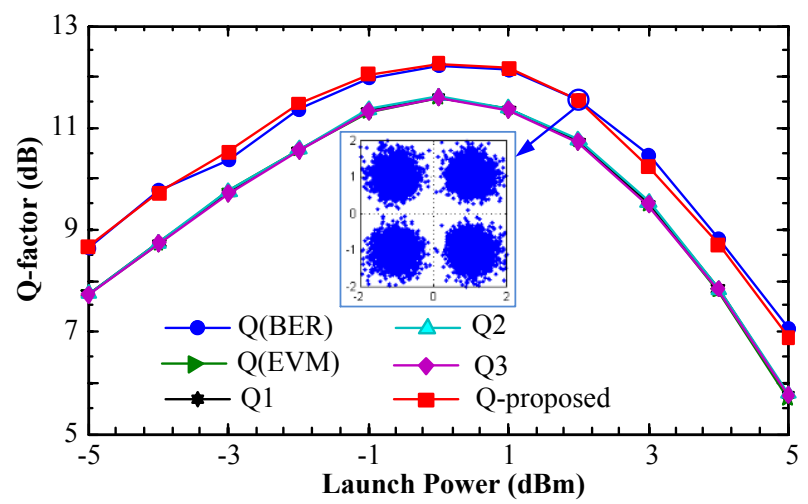

Fig. 7. Q-factor values (average over $\mathrm{x}$-and y-polarization) as a function of the launch power in $112 \mathrm{~Gb} / \mathrm{s}$ QPSK PDM CO-OFDM after $2400 \mathrm{~km}$ of transmission. Q(EVM), Q1, Q2, Q3 all follow the lower curve.

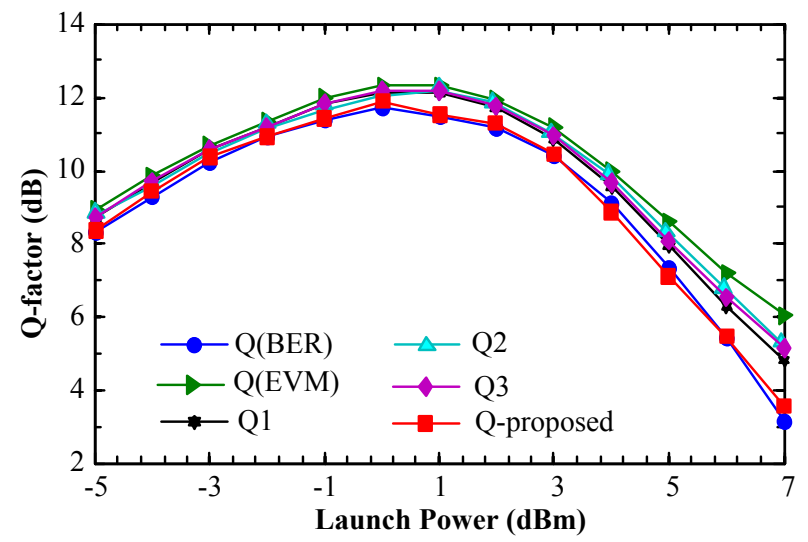

Fig. 8. Q-factor values (average over $\mathrm{x}$-and y-polarization) as a function of the launch power in $112 \mathrm{~Gb} / \mathrm{s}$ QPSK PDM RGI-CO-OFDM after $4000 \mathrm{~km}$ of transmission.

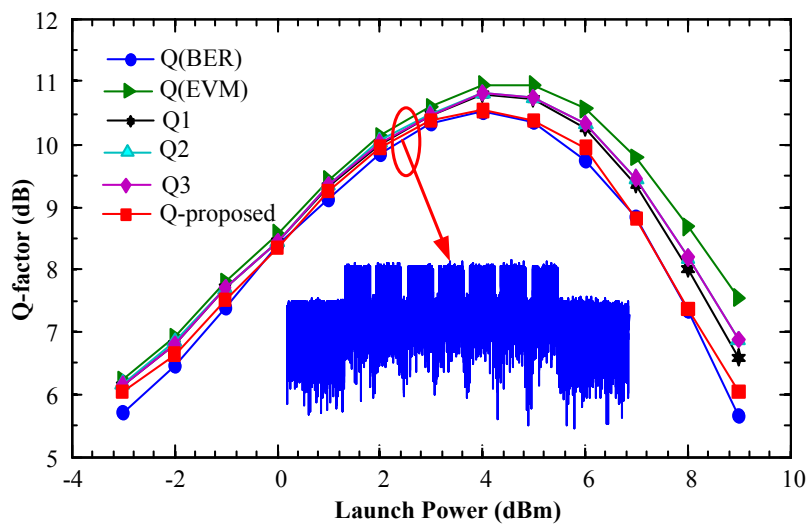

Fig. 9. Q-factor values for the center channel (average over $\mathrm{x}$-and $\mathrm{y}$ polarization) as a function of the launch power in $7 \times 112 \mathrm{~Gb} / \mathrm{s}$ QPSK PDM WDM RGI-CO-OFDM after $3200 \mathrm{~km}$ of transmission.
The performance of the BER estimation methods for $112 \mathrm{~Gb} / \mathrm{s}$ QPSK PDM RGI-CO-OFDM is shown in Fig. 8. In $112 \mathrm{~Gb} / \mathrm{s}$ QPSK RGI-CO-OFDM transmissions with OFDE at the receiver, the proposed BER estimation method also shows excellent agreement with the direct error counting result. Other BER estimation methods, unlike the case of the conventional CO-OFDM transmission, overestimate the system performance. These BER estimation methods all show similar performance and the estimation inaccuracy increases with the launch power. When the launch power is low (ASE limited regime) EVM (data-aided and nondata-aided), Q-factor 2, Qfactor 3 show good agreement with the direct error counting technique. However, at a high level of the launch power (the nonlinear limited regime) the inaccuracy in estimation is significant and increases proportionally with the launch power. A closer inspection reveals that among the BER estimation methods considered here the data-aided EVM has the poorest performance. However, the difference in performances of dataaided EVM and other methods is not significant.

The BER estimation methods applied in WDM CO-OFDM transmissions transmission are now investigated. We simulate $7 \times 112 \mathrm{~Gb} / \mathrm{s}$ PDM WDM RGI-CO-OFDM transmissions with $50 \mathrm{GHz}$ frequency spacing. In Fig. 9 the Q-factors obtained by using different BER estimation methods for the center channel are compared. The proposed BER estimation method also shows an excellent performance despite the nonlinear impairments from neighboring WDM channels. Similar to single channel PDM RGI-CO-OFDM transmission, all other BER estimation methods overestimate the system performance. The data-aided EVM also shows the worst performance and nondata-aided, Q-factor 2 and Q-factor 3 have a similar performance.

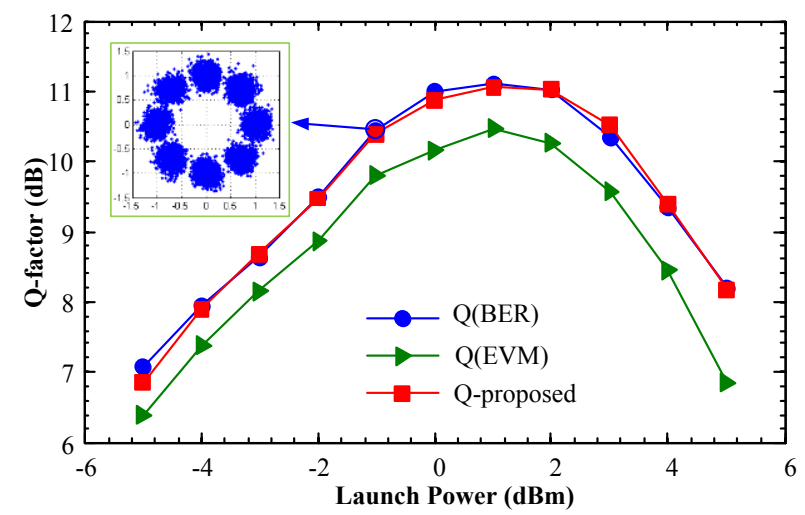

Fig. 10. Q-factor values (average over x-and y-polarization) as a function of the launch power in $112 \mathrm{~Gb} / \mathrm{s}$ 8-PSK PDM CO-OFDM after $800 \mathrm{~km}$ of transmission.

Figure 10 shows the performance of the proposed BER estimation method for $112 \mathrm{~Gb} / \mathrm{s}$ 8-PSK PDM CO-OFDM transmission. The estimation of the BER from data-aided EVM for 8-PSK systems can be can be found in [7]. As long as the received phases of each transmitted symbol has a Gaussian distribution, the proposed method also offers very accurate estimation of the system performance if m-PSK 
modulation format is adopted. However, the obtained results cannot be extended directly to high-order QAM modulation formats such as $8 \mathrm{QAM}$ or 16QAM, in which the decision is made by talking into account both the phases and amplitudes of the received symbols. For high-order QAM modulation formats, a 3D PDF would be required for estimating the system's BER. In this case, a larger number of statistical samples would be required for an accurate estimation which significantly increases the complexity of the estimator. This problem is beyond the scope of this paper. However, further investigation on this subject is of great interest.

\section{IMPACT OF THE LASER PHASE NOISE}

In this section we study the robustness of the proposed BER estimation method to laser phase noise. The laser phase noise can change the statistical properties of the received QPSK symbols, and thus affects the performance of all statistical BER estimation methods. For all results presented in this section the CPE is estimated and compensated using the pilotaided technique. For the conventional $112 \mathrm{~Gb} / \mathrm{s}$ CO-OFDM transmission 16 pilot subcarriers are inserted in each OFDM symbol, while for RGI-CO-OFDM the number of pilot subcarriers used is $6[25,26]$. Note that a smaller number of pilot subcarriers can be used in RGI-CO-OFDM due to the shorter symbol duration.

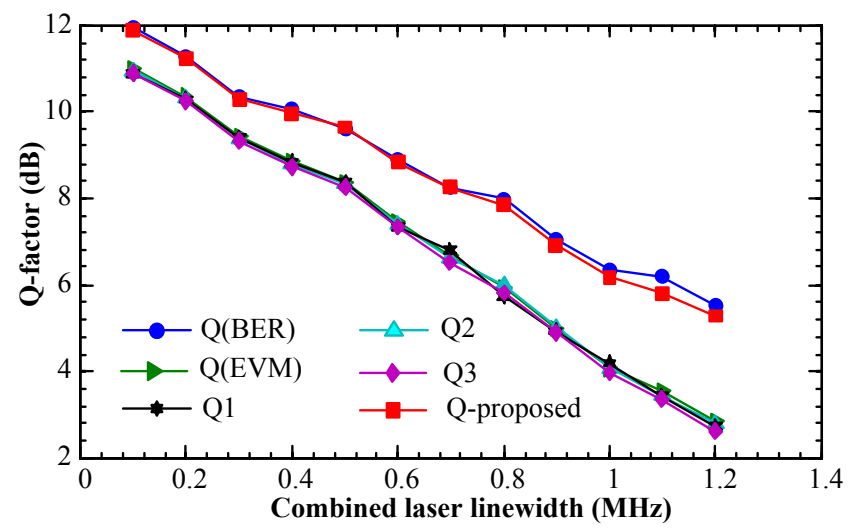

Fig. 11. Q-factor values (average over $\mathrm{x}$-and $\mathrm{y}$-polarization) as a function of the combined laser linewidth in $112 \mathrm{~Gb} / \mathrm{s}$ PDM CO-OFDM after $2000 \mathrm{~km}$ of transmission; the launch power was $1 \mathrm{dBm}$.

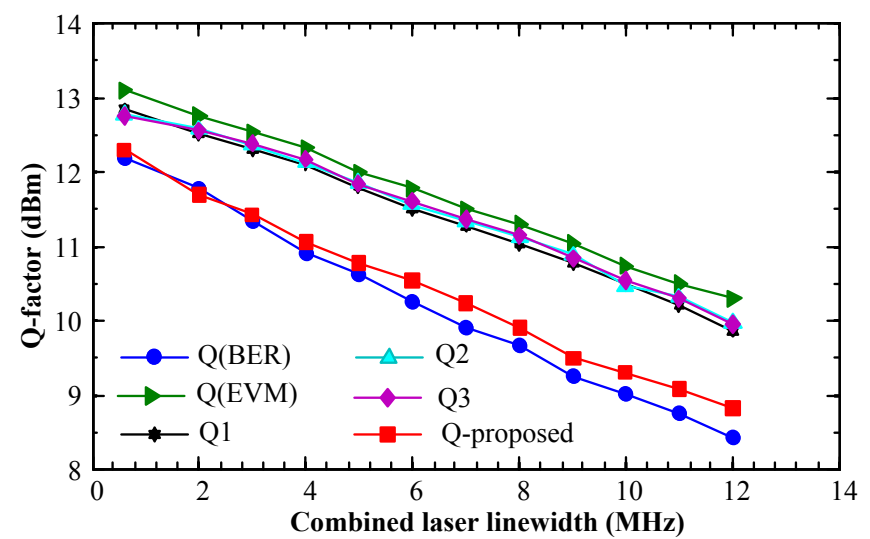

Fig. 12. Q-factor values (average over $\mathrm{x}$-and y-polarization) as a function of the combined laser linewidth in $112 \mathrm{~Gb} / \mathrm{s}$ PDM RGI-CO-OFDM after $4000 \mathrm{~km}$ of transmission; the launch power was $1 \mathrm{dBm}$.
The impact of laser phase noise on the performances of the BER estimation methods is shown in Fig. 11-12 for COOFDM and RGI-CO-OFDM transmissions. The combined laser linewidth is the sum of the linewidths of the transmitter and receiver lasers. We assume that the transmitter and receiver lasers have the same linewidth, which is equal to half of the combined linewidth. For $112 \mathrm{~Gb} / \mathrm{s}$ PDM CO-OFDM transmission almost no mismatch between the BERs estimated by the proposed method and the direct error counting was observed when the combined laser linewidth is increased up to 1.2-MHz. This result indicates that the proposed BER estimation method is extremely tolerant to laser phase noise. Note that commercial external-cavity lasers have a linewidth of around $100 \mathrm{kHz}$ which is the value used here. Other BER estimation methods, on the other hand, are much less tolerant to laser phase noise as their accuracy decreases when the combined laser linewidth is increased. At the combined laser linewidth of $200 \mathrm{kHz}$, all the aforementioned methods underestimate the system performance by approximately $1 \mathrm{~dB}$. However, if the combined laser linewidth is increased to 1.2 $\mathrm{MHz}$ the difference in Q-factor increases to over $2.5 \mathrm{~dB}$.

For $112 \mathrm{~Gb} / \mathrm{s}$ PDM RGI-CO-OFDM the combined laser linewidth is increased to $12 \mathrm{MHz}$ for investigating its impact on the performance of BER estimation methods. It can be seen that RGI-CO-OFDM is much more tolerant to laser phase noise in comparison with the conventional CO-OFDM because of the shorter symbol duration. In our investigated systems the symbol duration of RGI-CO-OFDM is $4 \mathrm{~ns}$, which is 18 times shorter than the symbol duration of the CO-OFDM system (72 ns). For the CO-OFDM system a $3 \mathrm{~dB}$ penalty due to laser phase noise is observed at $0.5 \mathrm{MHz}$ of combined laser linewidth while for RGI-CO-OFDM it occurs at $9 \mathrm{MHz}$ (also 18 times difference). As shown in Fig. 12, the proposed BER estimation method also shows an excellent tolerance towards the combined laser linewidth. Even though at high level of the combined laser linewidth this method also overestimates the system performance but the inaccuracy is relatively small, below $0.5 \mathrm{~dB}$ for $12 \mathrm{MHz}$ of the combined laser linewidth. On the other hand, for the same value of the combined laser linewidth, other BER estimation methods overestimate the system performance by around $3 \mathrm{~dB}$. Note that for RGI-COOFDM data aided EVM offers the worst performance while the combined laser linewidth is varied.

The results obtained in this section indicate that the proposed BER estimation method is highly tolerant to laser phase noise, both in CO-OFDM and RGI-CO-OFDM transmissions.

\section{IMPACT OF THE FREQUENCY OFFSET}

Similar to laser phase noise, the frequency offset between the transmitter and receiver lasers also changes the statistical properties of the received QPSK symbols. In CO-OFDM transmissions, this frequency offset can be effectively estimated and compensated using DSP techniques [27]. However, a residual uncompensated carrier frequency offset is always present in the system and thus the performance of 
statistical BER estimation methods may be affected. In this section we study the impact of carrier frequency offset on the effectiveness of the proposed BER estimation method.

For this investigation the frequency offset in CO-OFDM and RGI-CO-OFDM systems is varied up to $4 \mathrm{MHz}$ and $36 \mathrm{MHz}$ respectively. As the frequency spacing in RGI-CO-OFDM system can be much larger than that of the traditional COOFDM system, RGI-CO-OFDM is also more tolerant to the frequency offset. This is another major advantage of RGI-COOFDM as compared to traditional CO-OFDM systems.

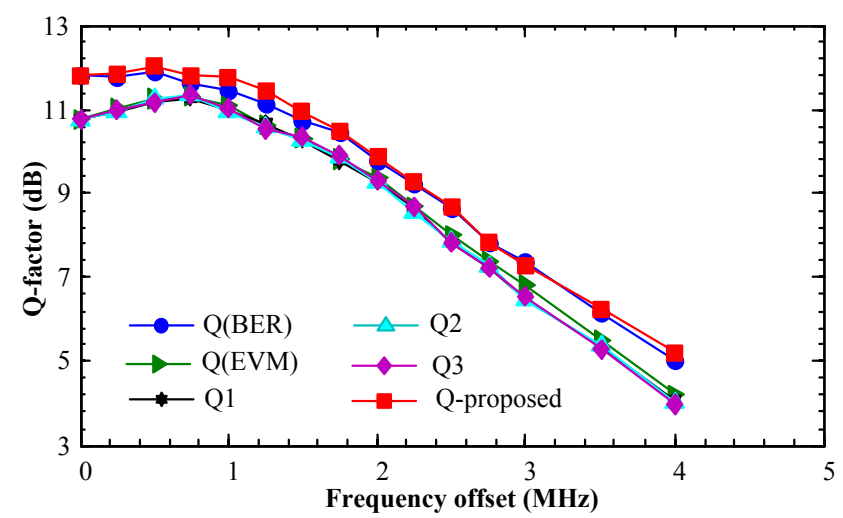

Fig. 13. Q-factor values (average over $\mathrm{x}$-and y-polarization) as a function of the frequency offset in $112 \mathrm{~Gb} / \mathrm{s}$ PDM CO-OFDM after $2000 \mathrm{~km}$ of transmission; the launch power was $1 \mathrm{dBm}$.

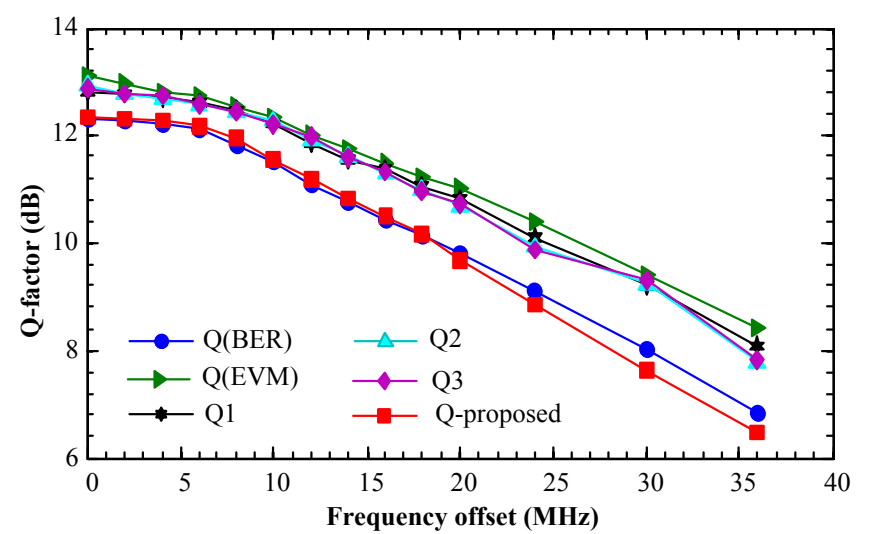

Fig. 14. Q-factor values (average over $\mathrm{x}$-and y-polarization) as a function of the frequency offset in $112 \mathrm{~Gb} / \mathrm{s}$ PDM RGI-CO-OFDM after $4000 \mathrm{~km}$ of transmission; the launch power was $1 \mathrm{dBm}$.

The simulation results are shown in Fig. 13 and Fig. 14. Remarkably, for both the conventional CO-OFDM and RGICO-OFDM systems the proposed BER estimation method also offers the most accurate estimation of the system performance in the presence of carrier frequency offset. However, some differences were observed for CO-OFDM and RGI-COOFDM transmissions. For RGI-CO-OFDM, the performance of all the BER estimation methods becomes worse if the frequency offset is increased. If the frequency offset is set to $36 \mathrm{MHz}$, the data-aided EVM overestimates the system performance by over $2 \mathrm{~dB}$ in comparison with $1 \mathrm{~dB}$ when no frequency offset is included. The proposed BER estimation method also underestimates the system performance in the presence of high frequency offset. However, the inaccuracy is insignificant. For CO-OFDM system, the proposed BER estimation method shows an excellent performance even in the presence of large frequency offset. Other BER estimation methods also offer good performances and their inaccuracies do not seem to increase with the frequency offset (up to $4 \mathrm{MHz}$ ). However, in general, the proposed BER estimation method shows a much better performance than other considered here techniques.

\section{CONCLUSION}

The statistical properties of CO-OFDM transmission are rather different from previously studied intensity modulation direct detection fibre-optic systems [28-32]. Therefore, new rules for BER estimates from numerical modelling have to be developed and verified. We have investigated the performance of a novel BER estimation method, which is based on the statistical properties of the received QPSK symbols, for COOFDM transmissions. Through numerical modeling of both the conventional PDM CO-OFDM and PDM RGI-CO-OFDM transmissions we demonstrate that this method is more accurate compared to commonly used BER estimators. In addition, we also show that the proposed BER estimation method is extremely tolerant to the laser phase noise and the frequency offset between transmitter and receiver lasers.

\section{ACKNOWLEDGMENT}

The support under the UK EPSRC Program Grant UNLOC $(\mathrm{EP} / \mathrm{J} 017582 / 1)$ is gratefully acknowledged.

\section{REFERENCES}

[1] W. Shieh and C. Athaudage, "Coherent optical orthogonal frequency division multiplexing," Electronics Letters, vol. 42, pp. 587-589, 2006.

[2] F. Buchali, R. Dischler, and X. Liu, "Optical OFDM: A promising high-speed optical transport technology," Bell Labs Technical Journal, vol. 14, pp. 125-146, 2009.

[3] A. J. Lowery, D. Liang, and J. Armstrong, "Orthogonal Frequency Division Multiplexing for Adaptive Dispersion Compensation in Long Haul WDM Systems," in Optical Fiber Communication Conference, 2006 and the 2006 National Fiber Optic Engineers Conference. OFC 2006, 2006, pp. 1-3.

[4] Z. Benyuan, D. Peckham, Y. Man, T. Taunay, and J. Fini, "Recent progress in transmission fibers for capacity beyond $100-\mathrm{Tbit} / \mathrm{s}$, " in Optical Fiber Communication Conference and Exposition (OFC/NFOEC), 2012 and the National Fiber Optic Engineers Conference, 2012, pp. 1-3.

[5] J. C. Cartledge, J. D. Downie, J. E. Hurley, Z. Xiaming, and I. Roudas, "Bit Error Ratio Performance of $112 \mathrm{~Gb} / \mathrm{s}$ PM-QPSK Transmission Systems," Lightwave Technology, Journal of, vol. 30, pp. 1475-1479, 2012.

[6] R. Schmogrow, B. Nebendahl, M. Winter, A. Josten, D. Hillerkuss, S. Koenig, et al., "Error Vector Magnitude as a Performance Measure for Advanced Modulation Formats," Photonics Technology Letters, IEEE, vol. 24, pp. 61-63, 2012.

[7] R. A. Shafik, S. Rahman, and R. Islam, "On the Extended Relationships Among EVM, BER and SNR as Performance Metrics," in Electrical and Computer Engineering, 2006. ICECE '06. International Conference on, 2006, pp. 408-411.

[8] H. Bao and W. Shieh, "Transmission simulation of coherent optical OFDM signals in WDM systems," Optics Express, vol. 15, pp. 44104418, 2007/04/16 2007.

[9] Z. Fan, L. Yazhi, W. Yandan, L. Li, Z. Lixin, C. Zhangyuan, et al., "Experimental Comparison of Different BER Estimation Methods for 
Coherent Optical QPSK Transmission Systems," Photonics Technology Letters, IEEE, vol. 23, pp. 1343-1345, 2011.

[10] G. Goldfarb and G. Li, "BER estimation of QPSK homodyne detection with carrier phase estimation using digital signal processing," Optics Express, vol. 14, pp. 8043-8053, 2006/09/04 2006.

[11] F. Zhang, Y. Gao, Y. Luo, Z. Chen, and A. Xu, "Stochastic BER estimation for coherent QPSK transmission systems with digital carrier phase recovery," Optics Express, vol. 18, pp. 9592-9599, 2010/04/26 2010.

[12] S. T. Le, K. J. Blow, V. K. Menzentsev, and S. K. Turitsyn, "Comparison of numerical bit error rate estimation methods in $112 \mathrm{Gbs}$ QPSK CO-OFDM transmission," in Optical Communication (ECOC 2013), 39th European Conference and Exhibition on, 2013, pp. 1-3.

[13] A. Carena, G. Bosco, V. Curri, P. Poggiolini, M. T. Taiba, and F. Forghieri, "Statistical characterization of PM-QPSK signals after propagation in uncompensated fiber links," in Optical Communication (ECOC), 2010 36th European Conference and Exhibition on, 2010, pp. 1-3.

[14] S. Randel, S. Adhikari, and S. L. Jansen, "Analysis of RF-Pilot-Based Phase Noise Compensation for Coherent Optical OFDM Systems," Photonics Technology Letters, IEEE, vol. 22, pp. 1288-1290, 2010.

[15] S. L. Jansen, I. Morita, T. C. W. Schenk, and H. Tanaka, "121.9-Gb/s PDM-OFDM Transmission With 2-b/s/Hz Spectral Efficiency Over $1000 \mathrm{~km}$ of SSMF," Lightwave Technology, Journal of, vol. 27, pp. 177-188, 2009.

[16] L. Xiang, S. Chandrasekhar, Z. Benyuan, P. J. Winzer, A. H. Gnauck, and D. W. Peckham, "448-Gb/s Reduced-Guard-Interval CO-OFDM Transmission Over $2000 \mathrm{~km}$ of Ultra-Large-Area Fiber and Five 80GHz-Grid ROADMs," Lightwave Technology, Journal of, vol. 29, pp. 483-490, 2011.

[17] R. Kudo, T. Kobayashi, K. Ishihara, Y. Takatori, A. Sano, and Y. Miyamoto, "Coherent Optical Single Carrier Transmission Using Overlap Frequency Domain Equalization for Long-Haul Optical Systems," Lightwave Technology, Journal of, vol. 27, pp. 3721-3728, 2009.

[18] M. Kuschnerov, F. N. Hauske, K. Piyawanno, B. Spinnler, A. Napoli, and B. Lankl, "Adaptive chromatic dispersion equalization for nondispersion managed coherent systems," in Optical Fiber Communication - incudes post deadline papers, 2009. OFC 2009. Conference on, 2009, pp. 1-3.

[19] S. J. Savory, "Digital filters for coherent optical receivers," Optics Express, vol. 16, pp. 804-817, 2008/01/21 2008.

[20] T. Xu, G. Jacobsen, S. Popov, J. Li, K. Wang, and A. T. Friberg, "Normalized LMS digital filter for chromatic dispersion equalization in 112-Gbit/s PDM-QPSK coherent optical transmission system," Optics Communications, vol. 283, pp. 963-967, 3/15/2010.

[21] P. Serena and A. Bononi, "On the accuracy of the Gaussian nonlinear model for dispersion-unmanaged coherent links," in Optical Communication (ECOC 2013), 39th European Conference and Exhibition on, 2013, pp. 1-3.

[22] Z. Wang, Y. Qiao, Y. Xu, and Y. Ji, "Statistical characterization of the nonlinear noise in $2.8 \mathrm{Tbit} / \mathrm{s}$ PDM-16QAM CO-OFDM system," Optics Express, vol. 21, pp. 18034-18042, 2013/07/29 2013.

[23] X. Chen and W. Shieh, "Closed-form expressions for nonlinear transmission performance of densely spaced coherent optical OFDM systems," Optics Express, vol. 18, pp. 19039-19054, 2010/08/30 2010.

[24] G. Gao, X. Chen, and W. Shieh, "Analytical Expressions for Nonlinear Transmission Performance of Coherent Optical OFDM Systems With Frequency Guard Band," Lightwave Technology, Journal of, vol. 30, pp. 2447-2454, 2012.

[25] L. Son Thai, T. Kanesan, E. Giacoumidis, N. J. Doran, and A. D. Ellis, "Quasi-Pilot Aided Phase Noise Estimation for Coherent Optical OFDM Systems," Photonics Technology Letters, IEEE, vol. 26, pp. 504-507, 2014

[26] S. T. Le, T. Kanesan, M. McCarthy, E. Giacoumidis, I. Phillips, M. F. Stephens, et al., "Experimental Demonstration of Data-dependent Pilot-aided Phase Noise Estimation for CO-OFDM," in Optical Fiber Communication Conference, San Francisco, California, 2014, p. Tu3G.4.

[27] T. M. Schmidl and D. C. Cox, "Robust frequency and timing synchronization for OFDM," Communications, IEEE Transactions on, vol. 45, pp. 1613-1621, 1997.
[28] E. W. Laedke, N. Goder, T. Schaefer, K. H. Spatschek, and S. Turitsyn, "Improvement of optical fibre systems performance by optimisation of receiver filter bandwidth and use of numerical methods to evaluate Q-factor," Electronics Letters, vol. 35, pp. 2131-2133, 1999.

[29] E. G. Shapiro, M. P. Fedoruk, and S. K. Turitsyn, "Numerical estimate of BER in optical systems with strong patterning effects," Electronics Letters, vol. 37, pp. 1179-1181, 2001.

[30] E. G. Shapiro, M. P. Fedoruk, and S. K. Turitsyn, "Direct modeling of error statistics at $40 \mathrm{Gbit} / \mathrm{s}$ rate in SMF/DCF link with strong bit overlapping," in Optical Fiber Communication Conference, 2005. Technical Digest. OFC/NFOEC, 2005, p. 3 pp. Vol. 1.

[31] B. Slater, S. Boscolo, T. Broderick, S. K. Turitsyn, R. Freund, L. Molle, et al., "Performance analysis of 20Gb/s RZ-DPSK non-slope matched transoceanic submarine links," Optics Express, vol. 15, pp. 10999-11007, 2007/08/20 2007.

[32] B. Slater, S. Boscolo, V. K. Mezentsev, and S. K. Turitsyn, "Comparative Analysis of BER Estimation Methods in Numerical Simulation of 40-Gb/s RZ-DPSK Transmission With In-Line SOAs," Photonics Technology Letters, IEEE, vol. 19, pp. 607-609, 2007.

Son T. Le (M.E.) received his Master of Engineering (M.E.) degree with highest distinction from Southern Federal University of Russia in 2012. He joined AIPT as a PhD student in January 2013. His research mainly focuses on coherent optical orthogonal frequency-division multiplexing (CO-OFDM), fiber nonlinearity mitigation techniques and visible light communication.

Keith J. Blow (PhD) studied at Cambridge University where he was awarded a BA in Physics and Theoretical Physics in 1978. He then joined the Theory of Condensed Matter Group of the Cavendish Laboratory and received a Ph.D. for studies on Deep Impurities in Semiconductors in 1981. He joined the optics division of BT Labs in 1981 and worked on the theory of nonlinear optical propagation effects in fibres, principally solitons. This work developed into optical switching and the first demonstration of soliton switching in non-linear optical loop mirrors. In $1990 \mathrm{He}$ set up a group working on quantum optical properties and non-linear spatial optics as well as continuing work on all-optical processing which is currently concerned with ways of using and manipulating the information that can be sent over the enormous bandwidth of optical fibres. In 1999 He moved to the Photonics Research Group at Aston to continue working on optical networks and optical computation. In 2003 he formed the Adaptive Networks Communications Research Group to study ad-hoc sensor networks, applications of dynamic hardware and mobile systems.

Vladimir Mezentsev $(\mathrm{PhD})$ received a $\mathrm{PhD}$ in 1991 from Institute of Automation, Russian Academy of Science, Novosibirsk Russia. His research interests include nonlinear waves, solitons and collapses, numerical modelling of nonlinear phenomena. He is currently a Senior Lecturer at Aston University in Birmingham where he joined in 1999. His recent research is focused primarily on femtosecond micro fabrication in photonics and also on high performance numerical modelling of fiber communication systems and ultrashort laser pulse propagation.

Sergei Turitsyn (Ph.D.) graduated from the Department of Physics of the Novosibirsk State University, Russia in 1982 and received his Ph.D. degree in Theoretical and Mathematical Physics from the Institute of Nuclear Physics, Novosibirsk, Russia in 1986. From 1992 to 1998 he worked in the Institute for Theoretical Physics I, Heinrich-Heine University Düsseldorf, Germany; first as a Humboldt Fellow and then within the collaborative projects with Deutsche Telekom. Professor Turitsyn is the director of the Aston Institute of Photonic Technologies at Aston University. He has made contributions to nonlinear science, including soliton theory, self-focusing of light beams, discrete nonlinear systems, and nonlinear fibre optics. His recent research has been shifted towards the high-speed optical communications, nonlinear photonic devices, Raman-based technologies, ultra-long fibre lasers and random fibre lasers. He was awarded the Royal Society Wolfson Research Merit Award in 2005, and was elected to OSA Fellow and AIP Fellow in 2011. 